\title{
An economical system for collecting and reducing heart-rate data, using someone else's small computer
}

\author{
CHARLES CLIFTON, JR. \\ University of Massachusetts, Amherst, Massachusetts 01002
}

Hardware and program are described for a heart-rate data collection and reduction system that uses a small computer at a time removed from the actual data collection. The system is portable and inexpensive; exclusive of the computer, it can cost under $\$ 1,500$. It uses an ordinary tape recorder to record discrete signals upon each occurrence of a heart beat, and it returns edited second-by-seccind averages of interheart-beat intervals.

Some researchers interested in heart-rate phenomena may find themselves in a situation in which they have a limited amount of equipment funds available, access to a small process control computer at its off hours, and a low tolerance for hand scoring of electrocardiographic (EKG) or cardiotachographic data. Such researchers may be interested in the following solution to their heart-rate data problems. The solution to be described has been implemented in three somewhat different manners for two investigators at the University of Masachusetts.

\section{HARDWARE}

The solution requires, as hardware, an EKG preamplifier or a cardiotachometer, an audio tape recorder of reasonable quality, a small amount of programming and pulse-forming equipment, and access to a small computer. The total cost of the hardware, exclusive of the computer, need not exceed $\$ 1,500$. The basic purpose of the system is to record on the audio tape recorder a discrete signal (a 1-msec square wave has been used successfully) each time a QRS complex occurs, and later to measure the time between these signals, edit out artifacts, and reduce the data, using the small computer.

A block diagram of the apparatus is shown in Fig. 1. In the implemented systems, the EKG preamplifier is a Hewlett-Packard $350-3200 \mathrm{~A}$, a Beckman 481 B followed by a $482 \mathrm{M} 8$, or a Datascope Carditron Model 650 . The Hewlett-Packard preamplifier feeds into a specially constructed filter and amplifier, consisting of an emitter-follower, followed by a phase shift oscillator with tunable frequency, followed by an emitter-follower, similar to that described by Brenner (1967). It is used to collect heart-rate data on infants in their first year of life. The Beckman amplifier feeds into an ordinary audio preamplifier (Realistic SA 100B) with no filter circuitry, which seems to be adequate for the adult EKG data gathered using it. The purpose of the amplifiers in both of these cases is to amplify the voltage output of the EKG preamplifier sufficiently to activate the Schmitt trigger (BRS Digibits ST 202). The output of the Schmitt trigger feeds through an inverter (BRS Digibits IN 201) into a one-shot (BRS Digibits OS 203), the output of which is passed through a limiting resistor into the auxiliary input of an ordinary audio tape recorder running at $3 \frac{3}{4}$ ips.
The limiting resistor has a resistance equal to approximately three times the input impedance of the tape recorder.

In the system using the Datascope preamplifier, the second-stage amplifier, the Schmitt trigger, and the one-shot have been eliminated from the path of the EKG signal. The Datascope preamplifier (in common with most cardiotachometers) contains a comparable Schmitt trigger and pulse former, used to trigger the sweep of the EKG record on its integral miniature oscilloscope. The output of the pulse former has been tapped, and put through a high-pass RC filter with a cut-off of $666 \mathrm{~Hz}$ (a $\$ 50$ modification performed by the Datascope Company). It is usable directly as an input to the audio tape recorder.

The signals triggered by the QRS complexes are put on Channel 1 of the tape recorder. Channel 2 is used to record information about the time of presentation of a stimulus, so that heart-rate data can be examined with respect to the point of stimulus presentation. A signal from the

\section{DATA COLLECTION}

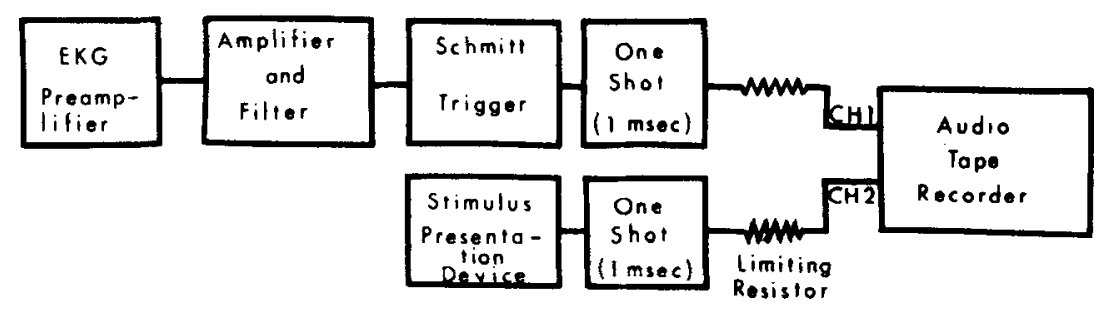

DATA REDUCTION

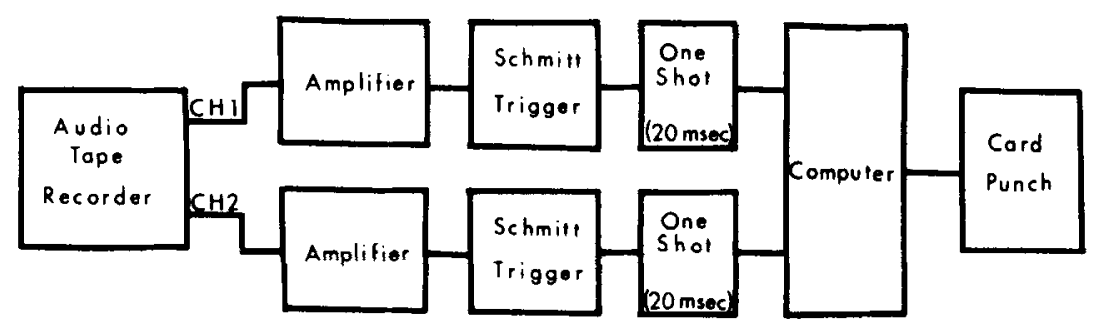

Fig. 1. Block diagram of the data collection and analysis apparatus. 
stimulus control apparatus (in the present implementations, systems of BRS Digibits or mixed Digibits and relay circuitry) is used to trigger a one-shot, whose output, fed through the proper resistance, is recorded on Channel 2 of the tape recorder.

The tape recorder that has been used in the present systems is a Revox $77 \mathrm{~A}$, a relatively expensive tape recorder but one which has an extremely constant tape-passing speed. If an investigator has little problem with fluctuations in line voltage or frequency, a less expensive tape recorder would be perfectly adequate.

The signals recorded on the tape are analyzed at some later time by being fed into a small computer, a PDP-8/I in the present case. The recorded tape is played into the computer at twice the speed at which it was recorded, to minimize data reduction time. The output of the audio tape recorder for each signal is a 1 -msec square wave, with some ringing if the record or playback level had been turned too high. This signal must be amplified to be fed into the computer being used currently. An ordinary stereo audio amplifier is perfectly satisfactory, although a "homemade" pair of transistor amplifiers is used in the present system. The amplified output of the signals corresponding to the QRS complexes is fed into one of two Schmitt triggers (DEC W 501), and the amplified output of the stimulus-presentation signal is fed into the other one. The output of each Schmitt trigger is fed into a one-shot with a 20-msec period, to inhibit false signals caused by ringing of the recorded signal or multiple signals placed on the tape by a QRS complex. The outputs of the one-shots are fed into a two-bit input buffer appropriate to the computer being used.

\section{PROGRAM}

A data-reduction program has been written in a combination of IL-8 (Headley, 1966) and PAL (Digital Equipment Corporation, 1970). 1 The program notes the occurrence of heart-beat and stimulus-presentation signals, records the absolute clock time of each occurrence, edits heart-rate artifacts in a manner to be described, computes weighted averages of the interheart-beat intervals during each second for a specifiable number of seconds prior to and following the occurrence of each stimulus presentation signal, and punches out the second-by-second averaged interbeat intervals for each trial on an IBM cardpunch.

The program first queries the computer operator regarding the number of trials (stimulus presentation signals or, in one version, a specifiable subset of the stimulus presentation signals) of data, the number of prestimulus seconds of reduced data he wants (Pre), the number of poststimulus seconds of data desired (Post), the shortest acceptable interbeat interval (lower limit), and the longest acceptable interbeat interval (upper limit). It then initiates data input from the tape recorder. A PAL subroutine identifies the occurrence of a heart-beat signal, and stores the absolute computer clock time (in milliseconds) of its occurrence in the top location of a pushdown store which contains the times of the three most recent beats. (This pushdown store was found to be necessary for infant data because of the slow operating speed of the main IL-8 program). The PAL subroutine also notes the time of occurrence of a stimulus signal.

The main IL-8 program places the absolute clock times at which nonartifactual heart-beat signals occurred into a separate 60-item pushdown store. It continuously checks locations in the PAL subroutine in order to determine when a heart-beat or stimulus presentation has occurred. When a heart-beat signal is noted, the interval that has elapsed since the previous heart-beat signal is calculated. If this interval is between the upper and the lower limits of interbeat intervals, which were specified earlier, then the time at which the just-noted heart-beat signal had occurred is put into the top location of a 60-item pushdown store. Artifactual heart-beat signals are rejected in two ways. First, any heart-beat signal that occurs too soon after an accepted beat is rejected. If the interval between the last heart-beat signal whose time of occurrence was stored in the IL-8 pushdown store and a just-noted heart-beat signal is less than the lower limit of interbeat intervals, the just-noted heart-beat signal is ignored. The computer waits for the next heart-beat signal and, when it arrives, calculates the interval between it and the time of the last beat that was stored in the IL-8 pushdown store to determine if the interval falls within the specified limits. Second, excessively long interbeat intervals are treated as though they should be filled by missing beats. If the interval between the last heart-beat signal stored in the IL-8 pushdown store and a just-noted heart-beat signal is greater than the upper limit of interbeat intervals, the program waits until two successive heart-beat signals arrive (the first of which may be the beat which ended the excessively long interval) whose calculated interbeat interval falls between the upper and lower limits. It then divides the interval between the last heart-beat time stored in the IL- 8 pushdown store and the first of these two acceptable beats into a whole number of equal intervals whose duration is as close as possible to the interval between these last two acceptable beats. These intervals are considered to be the reconstructed interbeat intervals for the missing beats. The times marking the divisions between the intervals (the reconstructed missing beat times), plus the last two acceptable true heart-beat times, are put into the top locations of the IL-8 pushdown store. This store, then, contains the absolute clock times at which the last 60 "good" beats occurred.

The IL-8 program also continuously checks the PAL subroutine for the occurrence of a stimulus-presentation signal. When one is noted, the IL-8 pushdown store of heart-beat times is examined to find the heart beat which occurred just prior to a time Pre sec before the time of occurrence of the stimulus-presentation signal. The program then calculates a weighted average (described below) of the interbeat intervals within each second from a time preceding the stimulus presentation by exactly Pre sec, through a time Post sec after the stimulus presentation. The calculation of these averages shares computer time with the identification, editing, and storing of additional heart-beat occurrence times.The program could be modified to obtain a specified number of interbeat intervals, for a beat-by-beat rather than a second-by-second analysis.

The method of calculation of the second-by-second data is to determine the value in milliseconds of each interbeat interval that falls within, or overlaps with, each successive second for which data are desired, multiply each such interbeat interval by the fraction of the second which that interval occupies, and obtain the sum of these products for each second. These sums, which are the weighted averages of the interbeat intervals for each second from Pre sec before the stimulus presentation to Post sec after the stimulus, are stored in a buffer with a capacity of 1,000 sec.

When the desired number of seconds of data have been computed following the last trial, the program outputs the stored averaged interbeat intervals on an IBM cardpunch. If an output device is available which can be run on-line with the data input (e.g., a magnetic tape recorder or a high-speed paper-tape punch), then the calculated data can be read out at the same time that new data are being read in. The final output, then, is a second-by-second record of edited 
averaged interbeat intervals, referred precisely to the time of occurrence of a stimulus. Careful checks of the output, which is appropriate for further analysis on a batch-processing computer, indicate that it is accurate to well within the limits of error in hand-seoring of EKG or cardiotachographic records.

\section{REFERENCES}

BRENNER, J. Heart rate, In P. Venables and I. Martin (Eds.), A manual of psychophysiological techniques. Amsterdam: North Holland, 1967.

DIGITAL EQUIPMENT CORPORATION Programming longuages. Maynard, Mass: Digital Equipment Corporation, 1970.

HEADLY, P. Interpreter language for the PDP.8. Ann Arbor, Mich: Mental Health Research Institute, University of Michigan, 1966.

\section{NOTE}

1. Paper-tape copies of the symbolic program are available from the author at the Department of Psychology, University of $M$ assachusetts, Amherst, Massachusetts 01002 , for the cost of preparing the copy. Users will also have to obtain a copy of the IL.8 assembler (Headly, 1966). Copies have been available from the Mental Health Reserach Institute of the University of Michigan. However, MHRI has implemented a revised version of the language, IL-8/I. which will necessitate some modifications in the program described here. In addition. modifications in the PAL subroutines will be necessary to adapt the program to a users' particulax computer configuration. 